\title{
Improving the Estimation of Uncalibrated Fractional Phase Offsets for PPP Ambiguity Resolution
}

\author{
Xingxing $\mathrm{Li}^{1,2}$ and Xiaohong Zhang ${ }^{1}$ \\ ${ }^{1}$ (School of Geodesy and Geomatics, Wuhan University, China) \\ ${ }^{2}$ (The German Research Centre for Geosciences (GFZ), Potsdam, Germany) \\ (E-mail: xhzhang@sgg.whu.edu.cn)
}

\begin{abstract}
Integer ambiguity resolution in Precise Point Positioning (PPP) can shorten convergence time and improve accuracy significantly. Uncalibrated Fractional Offsets (UFOs) originating in the satellites destroy the integer nature of carrier phase ambiguities observed at a single station. Several methods have been developed to estimate UFO information from a reference network for PPP ambiguity resolution. In this paper, we present a new approach for estimating Zero-Differenced (ZD) UFOs via float ZD ambiguities from a reference network. In this new approach, UFOs for receivers and satellites are estimated in an integrated adjustment with integer ambiguities being resolved sequentially, so that UFOs of higher quality can be achieved. The float ZD ambiguities used in the estimation can be from network or PPP solutions. Using those from PPP solutions enables the time-consuming clock estimations and the UFO estimations to be carried out separately, so that UFOs can be estimated more reliably from a much denser network. In this paper, a data processing procedure, from the estimation of UFOs through to PPP ambiguity fixing, is designed and proposed. The approach is validated with several data sets in various scenarios. The results show that satellite UFOs can be estimated precisely and reliably by using the observations from a reference network, in which the station spacing could be up to thousands of kilometres. With 30 minutes of observations, a PPP solution with fixed integer ambiguities is achievable and the positioning accuracy can be improved significantly when compared with its float solution.
\end{abstract}

\section{KEY WORDS}

1. Precise Point Positioning (PPP). 2. Zero-Difference (ZD) Integer Ambiguity Resolution. 3. Uncalibrated Fractional Offsets (UFOs).

First published online: 23 March 2012.

1. INTRODUCTION. Precise Point Positioning (PPP) has become a major research area in the Global Positioning System (GPS) community as it could provide an accurate positioning service at a large-scale without the support of dedicated reference stations (Bisnath and Gao 2007; Kouba and Héroux 2001). It has been demonstrated to be a powerful tool in geodetic and geodynamic applications (Zumberge et al., 1997; Kouba and Héroux 2001; Gao and Shen 2001; Zhang and Andersen 2006). However, traditional PPP needs a long convergence time to achieve centimetre-level accuracy, 
so its application in rapid and real-time positioning is presently limited (Bisnath and Gao 2007; Li et al., 2010).

In order to shorten the convergence time and improve accuracy, several approaches for fixing integer ambiguities in PPP solutions have been developed in recent years. In the approach by Gabor and Nerem (1999), Single-Differenced (SD) Uncalibrated Fractional Offsets (UFOs) between satellites are used for removing the fractional part in SD ambiguities at the user for integer ambiguity fixing, based on their simulations. Ge et al., (2008) demonstrated the high spatial and temporal stability of the satellite UFOs and presented an approach to estimate the fractional parts of the SD UFOs between satellites from a global reference network. Results showed an improvement of about $27 \%$ in the repeatability and $30 \%$ in the accuracy of the east component. Laurichesse et al., (2008) introduced another approach, in which 'integer' satellite clocks are estimated from a network, in order to fix the dualfrequency GPS ambiguities of undifferenced phase measurements. After applying such integer clocks in PPP, the resulting positioning precision is comparable to that of standard differential positioning. Collins et al., (2008) proposed a 'decoupled clock model, in which two sets of independent satellite clocks for phase and code respectively are used, requiring no assumption about the stability of code or phase biases. Although the above-mentioned approaches are realised in quite a different way, a comparative study (Geng et al., 2010) confirmed their theoretical equivalence; thus we concentrate on the approach using UFOs instead of 'integer' clocks.

The UFOs are usually derived from the estimated Zero-Differenced (ZD) ambiguities. In the approach by (Ge et al., 2008), all possible SD ambiguities are formed for each satellite pair. Their fractional parts should be statistically the same if the ZD ambiguities are estimated precisely, as further difference between any two of them forms a Double-Differenced (DD) ambiguity, which should be very close to an integer. By taking the mean of the fractional part of the SD ambiguities of the same satellite pair over all the stations, the SD UFO is estimated.

In this study, instead of estimating a SD UFO for all satellite pairs, we present a new approach; ZD UFOs are estimated in an integrated adjustment together with sequential integer ambiguity fixing in order to enhance the estimates. The approach can be applied to both Wide-Lane (WL) and Narrow-Lane (NL) combinations if the corresponding ZD ambiguities are available. It does not matter whether the NL ambiguities of the reference stations are derived from PPP or network modes. Using NL ambiguities from PPP enables the UFO to be estimated from a network that is much denser than that necessary for the time-consuming task of clock estimation (Zhang et al., 2010).

After a short introduction to the related mathematical background, the new approach is presented in detail. The algorithm for estimating UFOs from reference networks and applying them to a single station is described. Then, the new approach is evaluated for a variety of applications using several sets of data. The results of the validation are then presented and discussed.

2. ZD OBSERVATION MODEL. GPS ZD pseudo-range and carrier phase equations can be expressed as follows (Teunissen and Kleusberg, 1996):

$$
P_{i}^{k}=\rho+I_{i}^{k}+T_{i}^{k}+c\left[d t_{i}-d t^{k}\right]+c\left[d_{i}(t)+d^{k}\left(t-\tau_{i}^{k}\right)\right]+e_{i}^{k}
$$




$$
L_{i}^{k}=\rho-I_{i}^{k}+T_{i}^{k}+c\left[d t_{i}-d t^{k}\right]+c\left[\delta_{i}(t)+\delta^{k}\left(t-\tau_{i}^{k}\right)\right]+\lambda\left[\phi_{i}\left(t_{0}\right)-\phi^{k}\left(t_{0}\right)\right]+\lambda N_{i}^{k}+\varepsilon_{i}^{k}
$$

where:

superscript ' $\mathrm{k}$ ' refers to a given satellite.

subscript ' $i$ ' refers to the receiver.

$t$ is the time when the signal is received.

$\tau_{i}^{k}$ is the signal travel time between the satellite and receiver antenna phase centres.

$\rho$ is the geometric distance between the receiver and satellite antenna phase centres, at the signal reception and transmission times, respectively.

$I_{i}^{k}$ is the ionospheric delay on the path.

$T_{i}^{k}$ is the tropospheric delay on the path.

$c$ is the speed of light.

$d t_{i}$ is the receiver clock correction at the signal reception time.

$d t^{k}$ is the satellite clock correction at the time of signal transmission.

$d_{i}(t)$ is the signal delay from the receiver antenna to the signal correlator in the receiver.

$d^{k}\left(t-\tau_{i}^{k}\right)$ is the signal delay from the satellite signal's generation to its emission from the satellite antenna.

$e_{i}^{k}$ is the pseudo-range measurement noise.

$\delta_{i}(t)$ is the carrier phase signal delay from the receiving antenna to the signal correlator in the receiver.

$\delta^{k}\left(t-\tau_{i}^{k}\right)$ is the carrier phase signal delay from the satellite signal's generation to its transmission from the satellite antenna.

$\lambda$ is the wavelength.

$\phi_{i}\left(t_{0}\right)$ is the initial phase of the receiver at the reference time $t_{0}$.

$\phi^{k}\left(t_{0}\right)$ is the initial phase of the satellite at the reference time $t_{0}$.

$N_{i}^{k}$ is the integer ambiguity.

$\varepsilon_{i}^{k}$ is measurement noise of carrier phase observations.

By examining Equation (2), one can find that the float items of $\phi^{k}\left(t_{0}\right), \phi_{i}\left(t_{0}\right)$, $\delta^{k}\left(t-\tau_{i}^{k}\right), \delta_{i}(t)$ are also ambiguous. In the traditional standard PPP model (Kouba and Héroux 2001), these items will be treated as ambiguities. Therefore, the ambiguities in the traditional standard PPP model lose their integer property. They have to be estimated as float values. It is worthwhile noting that the choice of analysis strategy and algorithm in the traditional standard PPP model results in ambiguities being estimated as float values. In the next section, an Integer Ambiguity Resolution method on the ZD level will be developed.

3. ZD INTEGER AMBIGUITY RESOLUTION. As the initial phases and hardware delays in Equation (2) are not easily separated, we can group them as uncalibrated phase offsets: $b_{i}$ for the receiver and $b^{k}$ for the satellite (Blewitt 1989; Hatch 1996; Hofmann-Wellenhof et al., 1992). The non-dispersive items in Equations (1) and (2), such as geometric distance, troposphere etc., can be grouped together into 
$\rho_{g}$. Then Equations (1) and (2) can be simplified:

$$
\begin{gathered}
L_{i}^{k}=\rho_{i g}^{k}-I_{i}^{k}+\lambda\left(b_{i}-b^{k}\right)+\lambda N_{i}^{k}+\delta m_{i}^{k}+\varepsilon_{i}^{k} \\
P_{i}^{k}=\rho_{i g}^{k}+I_{i}^{k}+d m_{i}^{k}+c\left(d_{i}+d^{k}\right)+e_{i}^{k}
\end{gathered}
$$

where:

$$
\begin{gathered}
b_{i}=\phi_{i}\left(t_{0}\right)+\frac{c}{\lambda} \delta_{i}(t) \\
b^{k}=\phi^{k}\left(t_{0}\right)+\frac{c}{\lambda} \delta^{k}\left(t-\tau_{i}^{k}\right)
\end{gathered}
$$

and we define:

$$
B_{i}^{k}=N_{i}^{k}+b_{i}-b^{k}
$$

where:

$N_{i}^{k}$ is the integer ambiguity.

$b_{i}$ is the receiver-dependent uncalibrated phase delay.

$b^{k}$ is the satellite-dependent uncalibrated phase delay.

$B_{i}^{k}$ is the comprehensive ambiguity without an integer property.

By examining Equation (7), one can find that the ZD ambiguity is contaminated by $b$. Generally, $b$ is assumed to be slowly varying with time. However, the ambiguity $B$ is considered as a constant and the receiver clock offset varies from epoch to epoch in GPS data processing. Consequently, the time-varying portion of $b$ will be mostly absorbed by the clock and the constant portion of $b$ will be absorbed into the ambiguity $B$. In addition, as code measurements are introduced into the PPP model, the group delay biases $d$ will also contribute to the ambiguity estimation. Generally, group delay biases $d$ vary slowly enough to be considered as constant over a short time interval (Gabor and Nerem, 1999). Therefore, contributions from $b$ and $d$ to the ambiguity could be assumed as float constant offsets in a short time interval. The constant offsets have an integer part and a fractional part. The integer part is difficult to separate from the original ambiguity $N$. Fortunately, the integer part will not make the ambiguity lose its integer property. The integer property of ZD ambiguity is destroyed by the fractional portion (i.e., the UFOs). To enable Integer Ambiguity Resolution on ZD observations, UFOs need to be separated from $B$.

The following part will show the method used to separate UFOs from $B$ in order to recover the integer property of the ZD ambiguity. We can reform Equation (7) as follows:

$$
B_{i}^{k}=\tilde{N}_{i}^{k}+f_{i}-f^{k}
$$

where:

$B$ denotes float ZD ambiguities in the standard model.

$\tilde{N}$ denotes the sum of $N$ and the integer part of $b$.

$f_{i}$ denotes the UFO of the receiver.

$f^{k}$ denotes the UFO of the satellite. 
In order to remove ionospheric delays, ionosphere-free observations (signal frequency L3) are usually used for the estimation of orbits and clocks and for PPP on the user side. The ambiguity of ionosphere-free combined observation (L3) can be expressed as follows:

$$
\begin{aligned}
\lambda_{L 3} \cdot B_{L 3} & =\frac{c f_{L 1}}{f_{L 1}^{2}-f_{L 2}^{2}} \cdot B_{L 1}-\frac{c f_{L 2}}{f_{L 1}^{2}-f_{L 2}^{2}} \cdot B_{L 2} \\
& =\frac{c f_{L 2}}{f_{L 1}^{2}-f_{L 2}^{2}} \cdot\left(B_{L 1}-B_{L 2}\right)+\frac{c}{f_{L 1}+f_{L 2}} \cdot B_{L 1} \\
& =\frac{c f_{L 2}}{f_{L 1}^{2}-f_{L 2}^{2}} \cdot B_{W L}+\lambda_{N L} \cdot B_{L 1}
\end{aligned}
$$

where:

$\lambda_{L 3}$ is the wavelength of ionosphere-free combination $(\sim 6 \mathrm{~mm})$.

$B_{L 3}$ is the ambiguity of ionosphere-free combination.

$c$ is the speed of light in a vacuum.

$f_{L 1}$ and $f_{L 2}$ refer to the signal frequencies of $\mathrm{L} 1$ and $\mathrm{L} 2$.

$B_{L 1}$ and $B_{L 2}$ are the ambiguities of $\mathrm{L} 1$ and $\mathrm{L} 2$.

Therefore, ambiguity resolution has to be conducted first in Wide-Lane (WL), and then in Narrow-Lane (NL). An L3 ambiguity is fixed as soon as both the WL and NL ambiguities are fixed. The WL ambiguity $B_{W L}$ can be calculated simply by taking the time average of the 'M-W' (Melbourne and Wübbena) combination (Melbourne 1985, Wübbena 1985) of the dual-frequency phase and range observations. The NL ambiguities are derived from Equation (9) with the already fixed WL ambiguity and the L3 ambiguity of the float ionosphere-free solution.

To recover the integer nature of ambiguities at a single station, the WL and NL UFOs must be estimated from the reference network and then provided to users as soon as the ZD ambiguities of the reference network are available.

4. ESTIMATION OF THE ZD UFOS. Let us assume that we have a network of ' $n$ ' stations tracking ' $m$ ' satellites. The float $Z \mathrm{D}$ ambiguities at each station are estimated as $b_{i}$. For each ZD ambiguity we have an observation Equation (10) in the form of Equation (8):

$$
\left[\begin{array}{c}
b_{1} \\
b_{2} \\
\vdots \\
\vdots \\
b_{n}
\end{array}\right]=\left[\begin{array}{ccccccc}
I & & & & R_{1} & S_{1} \\
& I & & 0 & & R_{2} & S_{2} \\
& & \vdots & & & & \\
& 0 & & I & & & \\
& & & I & R_{n} & S_{n}
\end{array}\right]\left[\begin{array}{c}
n_{1} \\
n_{2} \\
\\
n_{n} \\
f_{r} \\
f^{s}
\end{array}\right]
$$

where:

$n_{i}$ is the ZD integer ambiguity vector for station $\mathrm{i}$.

$f_{r}$ and $f^{s}$ are the UFOs for receivers and satellites respectively.

$R_{i}$ and $S_{i}$ are the coefficient matrices for receiver and satellite UFOs respectively.

$\mathrm{Q}$ is the covariance matrix of the $\mathrm{ZD}$ float ambiguities. 
In matrix $R_{i}$ all elements of one column are 1 and all other entries are zero. For matrix $S_{i}$ each line has one element of -1 , the other entries are zero.

Obviously, we cannot estimate the integer ambiguities and the UFOs simultaneously because the number of parameters are $m+n$ more than the number of pseudo-observations. Even if we knew all the integer ambiguities, there would still be a rank deficiency of 1 . That means we have to fix one of the UFOs to, for example, zero.

Under the condition that all the integer ambiguities are known exactly and that one UFO is fixed to zero, then the UFOs can be estimated by means of a least square adjustment from the following observation Equation (11):

$$
\left[\begin{array}{c}
b_{1}-n_{1} \\
b_{2}-n_{2} \\
\vdots \\
\vdots \\
b_{n}-n_{n}
\end{array}\right]=\left[\begin{array}{cc}
R_{1} & S_{1} \\
R_{2} & S_{2} \\
\vdots & \vdots \\
\vdots & \vdots \\
R_{n} & S_{n}
\end{array}\right]\left[\begin{array}{c}
f_{r} \\
f^{s}
\end{array}\right], \mathrm{Q}, \text { with } \mathrm{Q}\left(f_{r}(1)\right)=>0
$$

The ZD ambiguities must be estimated very precisely, so that their DD ambiguities are very close to integers. Otherwise, they cannot be used to derive UFOs. We assume that the UFO at the first arbitrarily selected station is zero. Then the nearest integers of the ZD ambiguities at this station are the integer ambiguities and the fractional parts are estimates of the corresponding satellite UFOs. When applying these satellite UFOs to the common satellites of the next station, the corrected ZD ambiguities should have a very similar fractional part. Of course, we can take the fractional part of one satellite as the UFO of the receiver. However, the mean fractional parts of all the common satellites, with proper quality control, give a better estimate of the receiver UFO. With this UFO, UFOs of the newly appearing satellites at the station can be estimated. Repeating this procedure for all stations, we can obtain the approximate UFOs for all receivers and satellites.

After correcting the ZD ambiguities with the UFOs, they should be very close to integers, thus ambiguity-fixing can be attempted. Replacing integer ambiguity parameters with their fixed values in Equation (10), the remaining parameters can be estimated. The UFO estimates are improved and will in turn help to resolve more integer ambiguities. The above procedure can be done iteratively until no more integer ambiguities can be fixed. The UFOs from the last iteration form the information needed by the user side for PPP ambiguity fixing.

The number of the fixed ambiguities contributing to a UFO parameter can be a very efficient indicator for use in quality control. From Equation (8), if an ambiguity cannot be fixed to an integer, the corresponding observation will not contribute to the estimation of UFOs at all. Therefore, satellite UFOs with contributions from few stations should not be disseminated to users, although the strategy does not require that all ambiguities must be fixed.

Based on the above UFO estimation approach, we developed a computational procedure for the estimation of UFOs on the server side and for using the UFOs for PPP on the user side. These are described in detail below.

First, we carry out a PPP float solution with International GNSS Service (IGS) orbit and clock products for all stations of the reference network with fixed station coordinates. The float WL ambiguities are estimated from the MW combination and 
the ionosphere-free ambiguities are estimated from the PPP solution. Then the approach in Section 4 is applied to all the float ZD WL ambiguities, so that the WL UFOs and integer ZD WL ambiguities are estimated. With the integer WL ambiguities estimated, the NL ambiguities are derived from the ionosphere-free ambiguities. Afterwards, the same approach is used to estimate the NL UFOs from the float NL ambiguities. Finally, we can offer the estimated WL and NL UFOs of satellites to PPP users together with IGS orbit and clock products, so that PPP with integer ambiguity fixing can be performed at a single station. The float WL and NL ambiguities are estimated in the same way as for the reference stations. Their satellite UFOs are removed by the estimated corrections, whereas the receiver UFO can be assimilated into the receiver clock by forcing one ZD ambiguity to its nearest integer. Afterwards, the ZD ambiguities have an integer property, so they can be fixed by making use of the same methods for fixing DD-ambiguities, such as the sequential ambiguity-fixing strategy (Dong and Bock, 1989) or the LAMBDA method (Teunissen, 1995). The detailed algorithm procedure, including the estimation of UFOs on the server side and PPP with integer ambiguity fixing on the user side, is shown in Figure 1.

In the above UFO estimation method, the estimated UFO will change slowly with time due to the inaccurately modelled atmospheric delays and the multipath effect, etc. The NL UFO estimation is more likely to be adversely affected because of its short wavelength. Although pseudo-range noises will be introduced into the WL ambiguity estimation, WL UFOs are often more stable over time because the WL combination has a relatively long wavelength. Generally speaking, WL UFOs can be estimated every day and used for real-time applications with a long update interval. On the other hand, NL UFOs need regular estimation with short update intervals (especially for receiver UFOs). For example, the method can estimate a group of UFOs every few minutes (10 minutes for all examples in this paper), and make short-term predictions of NL UFOs for real-time applications. We used the observations from a tracking network shown in Figure 4 during Day Of Year (DOY) 121-130 (30 Apr-9 May) in 2008 to estimate WL UFOs every day and NL UFOs every 10 minutes as shown in Figures 2 and 3, in which all 144 groups in day 122 are shown. One can find that WL UFOs are rather stable and can be predicted for a long time. NL UFOs are also relatively stable in the short term but can only be predicted for a short time.

5. VALIDATION EXPERIMENTS. In this section, to validate the proposed method, examine its feasibility, and evaluate positioning accuracy, we conducted experiments at stations both in static and kinematic positioning modes and the float and fixed solutions were then compared.

5.1. Experimental Deployment and Data Processing Strategy. We used three networks: a local Chengdu 'Continuous Operation Reference Station' (CORS) network with an average station spacing of $40 \mathrm{~km}$, the regional network of China with an average station spacing of $1200 \mathrm{~km}$, and a continental network of IGS tracking stations with an average station spacing of $2500 \mathrm{~km}$. These networks were used by the server to compute the UFOs to be applied to correct the ZD carrier phase measurements at the user. In the following sections, the distance between the PPP user and the network refers to the distance from the user to the closest station of the network. We name this the user-to-network distance. The three experiments with 


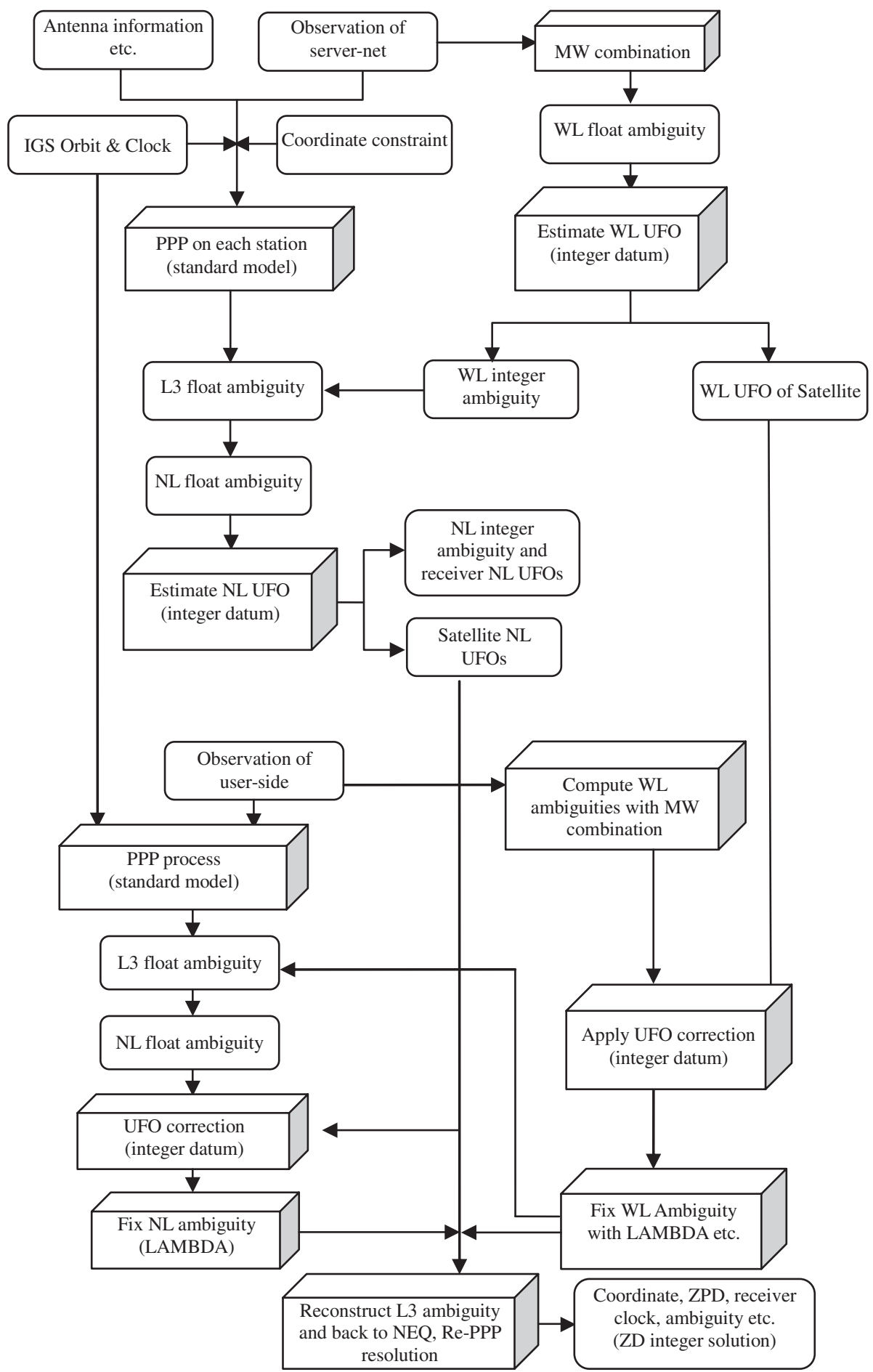

Figure 1. Data processing procedure for estimation of UFOs from a reference network and their application to a single station for PPP with integer ambiguity fixing. 


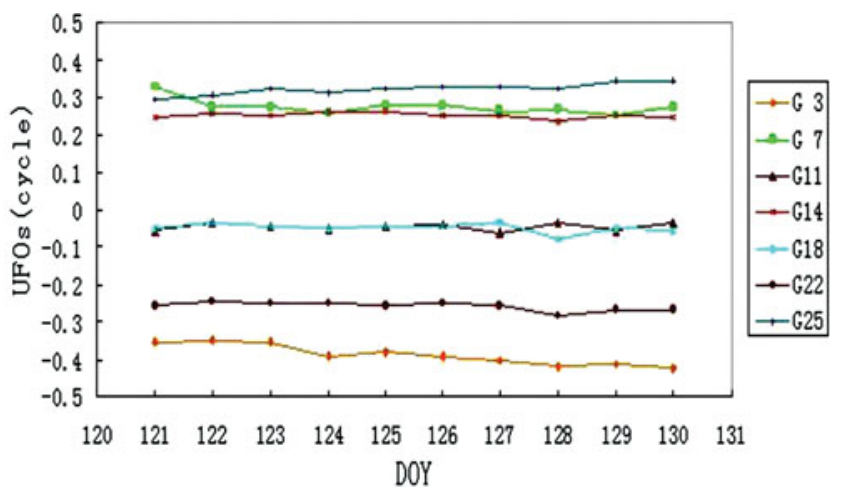

Figure 2. WL UFOs of satellites.

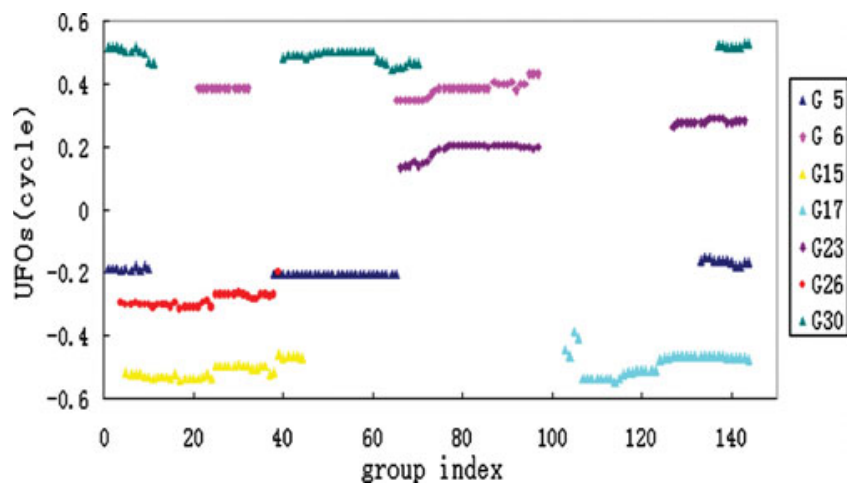

Figure 3. NL UFOs of satellites.

differently sized networks will be used to investigate the influence of network station density on the ZD ambiguity fixing rate and positioning accuracy. The details of these networks are described as follows:

- Experiment A. Stations inside China were chosen to make up the server-end of the observation network. The location of the network stations is shown in Figure 4, in which the ten blue solid circles represent the stations used to compute satellite UFOs. These stations are distributed approximately evenly across China with an average station spacing of about $1200 \mathrm{~km}$. The green solid triangles represent the PPP user stations, of which three stations are located inside the network and nine stations are outside the network, in order to test the feasibility and accuracy of the method proposed in this paper. The designed user-tonetwork distance ranges from $800 \mathrm{~km}$ to $3000 \mathrm{~km}$.

- Experiment B. IGS tracking stations outside China were chosen to make up the server-end of the observation network. The distribution of the network stations is shown in Figure 5, in which the ten blue solid circles represent the stations used to compute satellite UFOs. These stations are distributed from latitudes $14^{\circ}$ North to $68^{\circ}$ North and from longitudes $5^{\circ}$ East to $142^{\circ}$ East. Average station spacing is 


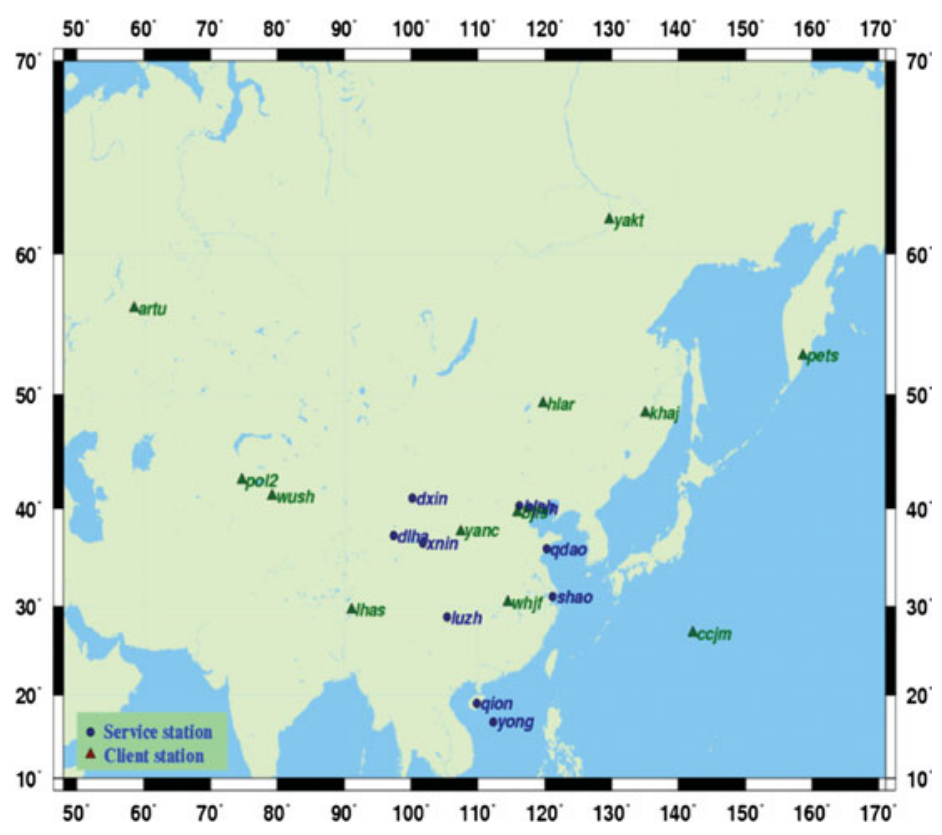

Figure 4. Network deployment of Experiment A.

about $2500 \mathrm{~km}$. The green solid triangles represent the PPP user stations to test the feasibility and accuracy of the method. The designed user-to-network distance for this experiment ranges from $800 \mathrm{~km}$ to $2500 \mathrm{~km}$.

- Experiment $C$. The local Chengdu CORS network was chosen to make up the server-end of the observation network. As CORS networks already exist in many cities worldwide, we designed this experiment to test the feasibility of augmenting PPP with CORS, which would supplement the shortage of existing stations in DD RTK networks (in which RTK services are only available within an area around tens of kilometres from the network). The distribution of the network stations is shown in Figure 6, in which the six blue solid circles are the Chengdu CORS stations used to compute satellite UFOs. Average station spacing is about $40 \mathrm{~km}$. The green solid triangles represent the PPP user stations used to test the feasibility and accuracy of the method. The designed user-to-network distance for this experiment ranges from $1200 \mathrm{~km}$ to $3500 \mathrm{~km}$.

Observation data from GPS week \#1477 in 2008 was used for the three experiments. Precise orbit and clock products were downloaded from the IGS website. On the service-end, a set of WL UFOs was estimated every day, while a set of NL UFOs was computed every 10 minutes. The observations from user stations were separated into sessions of 30 minutes. PPP fixed solutions and float solutions were then conducted in static mode. IGS products (SINEX and TRO files) were taken as the truth for comparison. The LAMBDA method was employed to calculate the ZD integer ambiguity, and partial ambiguity resolution technology (Teunissen et al., 1999) was applied to fix as many ambiguity parameters as possible if the ambiguity parameters could not be fully fixed. 


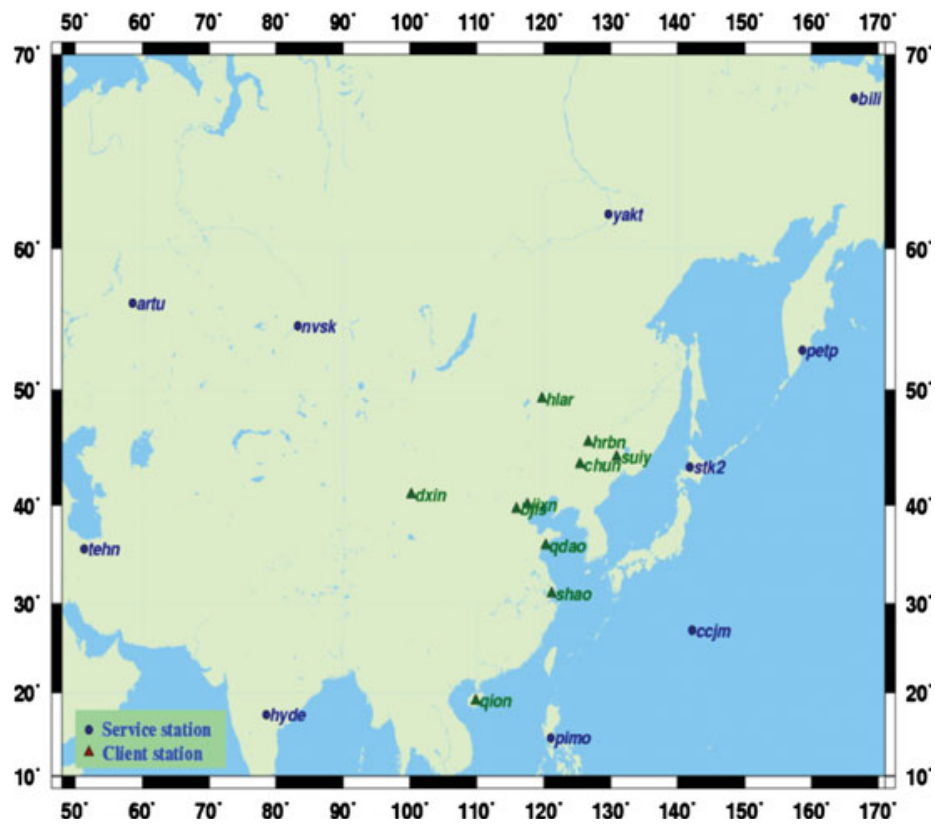

Figure 5. Network deployment of Experiment B.

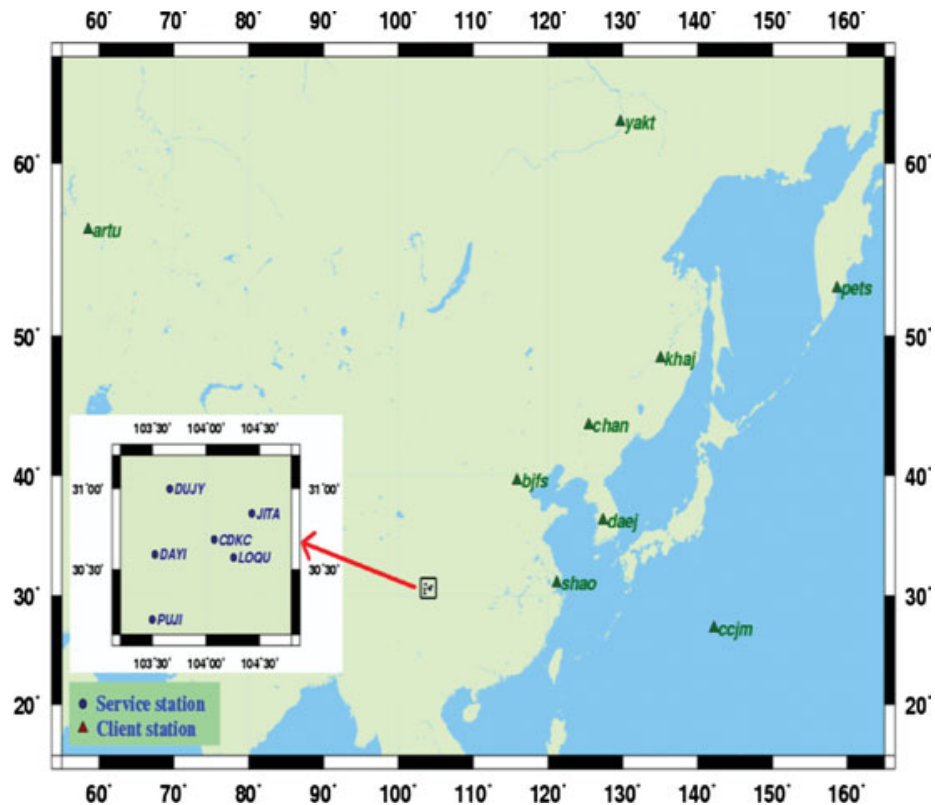

Figure 6. Network deployment of Experiment C.

5.2. Results and Analysis of Static Experiments. Statistical analysis was carried out on the results of Experiment A, including the positioning and Zenith Path Delay (ZPD) results of the float and fixed solutions and the success rate of the fixed solution 


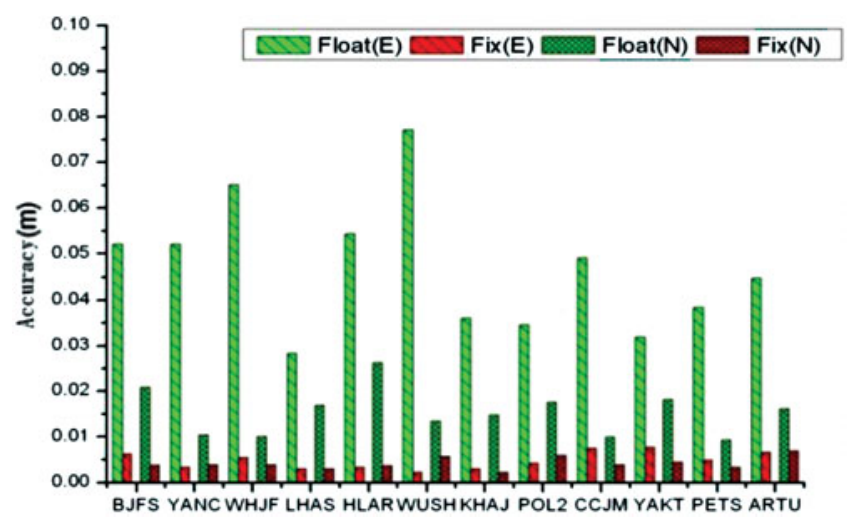

Figure 7. Horizontal accuracy of Experiment A.

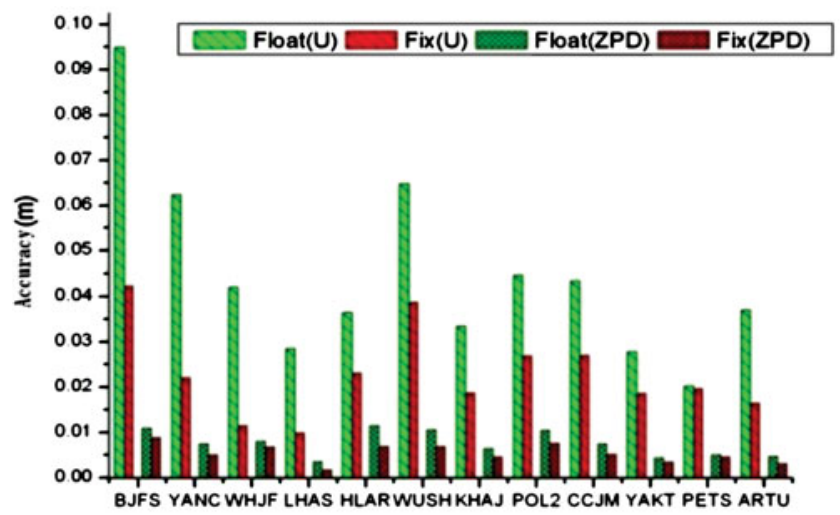

Figure 8. Height and ZPD accuracy of Experiment A.

(the ratio of the number of successfully fixed segments to the total number of segments). With thirty minutes of observation data, the positioning accuracy (the mean value of the absolute errors of all the time segments on a station) in Easting (E), Northing (N) and Vertical (U) directions and the ZPD accuracy of the PPP float and fixed solutions respectively are shown in Figure 7 and Figure 8.

When examining Figure 7 and Figure 8, one can see that, with thirty minutes of static data, the positioning accuracy of the float solution is $1 \sim 3 \mathrm{~cm}$ in the North component, $3 \sim 8 \mathrm{~cm}$ in East component and $3 \sim 10 \mathrm{~cm}$ in vertical, while the positioning accuracy of the fixed solution is improved to the millimetre level (usually within $5 \mathrm{~mm}$ ) horizontally and about $1 \sim 4 \mathrm{~cm}$ vertically. Moreover, the improvement by the fixed solution on the east component is much more notable. In Figure 8, it can be found that the ZPD accuracy of the fixed and float solutions are generally within $1 \mathrm{~cm}$, while the fixed solution ZPD was improved by 10-30\% when compared with the float solution. Some stations (such as 'LHAS' and 'HLAR') even improved by $50 \%$. According to statistical results, the fixing success rate for stations away from the network, but within $2000 \mathrm{~km}$, is nearly $100 \%$, while that for user stations away from 


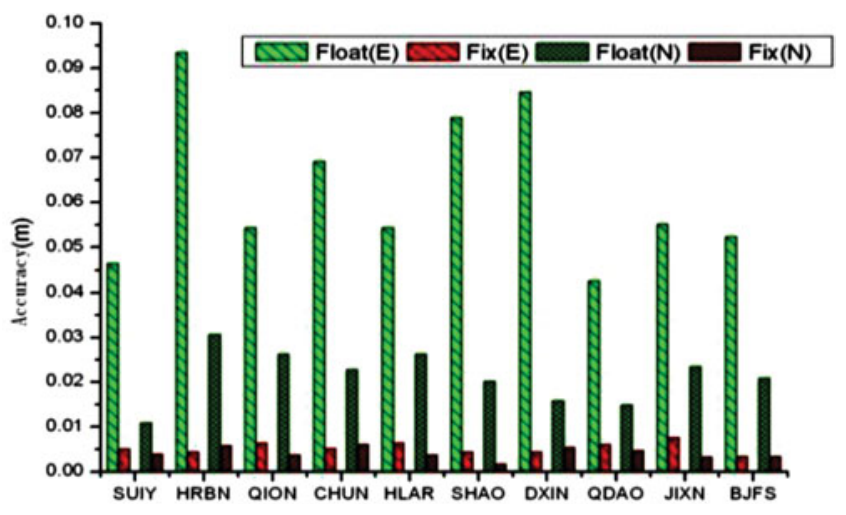

Figure 9. Horizontal accuracy of Experiment B.

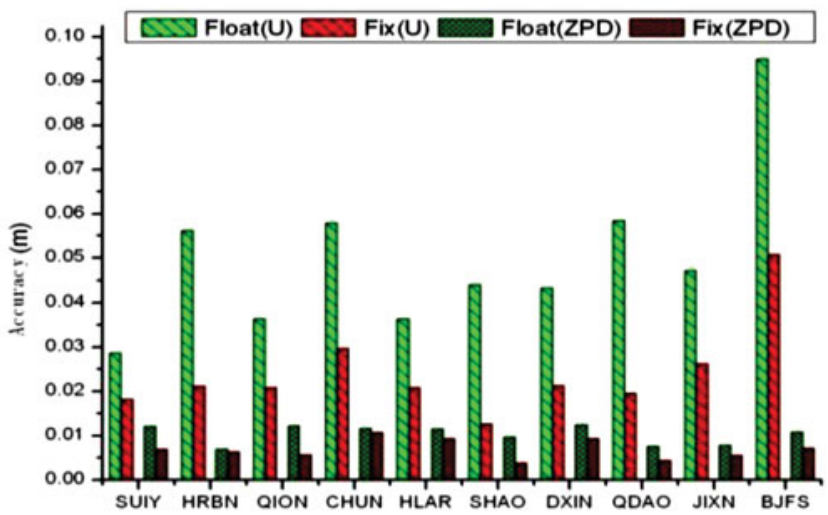

Figure 10. Height and ZPD accuracy of Experiment B.

the network over $2000 \mathrm{~km}$ is slightly lower but still above $90 \%$. This shows that the UFO products estimated by ten service stations in China can provide high-accuracy rapid GPS positioning and a meteorology service for PPP users within an area up to about $3000 \mathrm{~km}$ of the network.

Based on the same analysing strategy used above, similar statistical analysis was carried out for Experiments B and C. Results are shown in Figures 9 to 12.

When examining Figures 9 to 12, similar to those of Experiment A, the positioning accuracy of the fixed solution improved significantly compared to that of the float solution. From Figures 10 and 12, one can find that the ZPD accuracy of both the fixed and float solutions are generally within $1 \mathrm{~cm}$, and the fixed solution ZPD improves by $10-50 \%$ compared to the float solution. According to our definition of fixing success rate, the success rates for most stations in the network are close to $100 \%$; the stations away from the network but within $2500 \mathrm{~km}$ usually have a success rate of above $90 \%$. The fixing success rate of stations over $3000 \mathrm{~km}$ away from the network is still above $80 \%$. This is probably because the user station is too far away from the service network and hence some satellites that are observable from the user station cannot be observed at the tracking stations. Experiment B demonstrates that sparsely distributed IGS tracking stations positioned outside China, with an average spacing of 


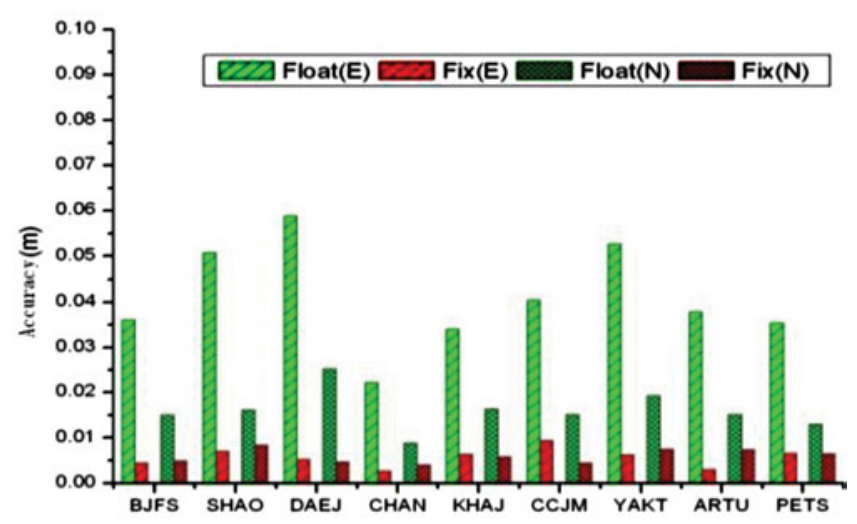

Figure 11. Horizontal accuracy of Experiment C.

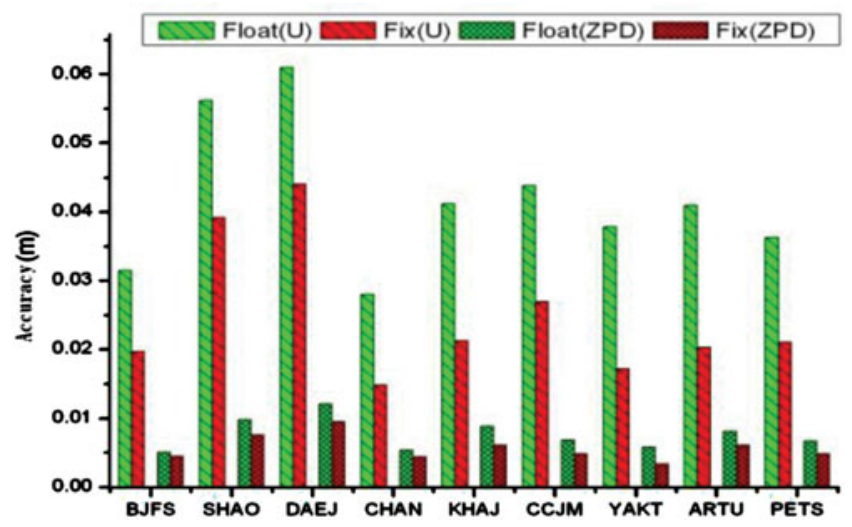

Figure 12. Height and ZPD accuracy of Experiment C.

about $2500 \mathrm{~km}$, are already sufficient for reliable, high-accuracy, and rapid positioning as well as meteorological services for the whole country. Experiment $\mathrm{C}$ illustrates that UFOs derived from a regional network such as the Chengdu CORS network can also provide reliable and high-accuracy service for PPP users almost countrywide.

5.3. Kinematic Experiment. The GPS data collected on 12 May 2008 (around the Mw 8.0 Wenchuan earthquake in Sichuan Province, China) was selected for the task of retrieving the surface deformation induced by the earthquake. The observations at stations 'BANA' and 'HECU', which are about $350 \mathrm{~km}$ away from the epicentre, were processed in kinematic mode. The results derived from the observations from thirty minutes before to thirty minutes after the earthquake are presented in Figure 13 and Figure 14. The figures on the left show the displacement time series of the float solution while figures on the right show the fixed PPP solution. In the figures, the letters $\mathrm{N}, \mathrm{E}$ and $\mathrm{U}$ denote the north, east and up components respectively. Other stations produced similar results to these two.

As we can see from Figure 13(a) and Figure 14(a), displacements derived from the float solution are comparatively stable in the North direction with an accuracy better 


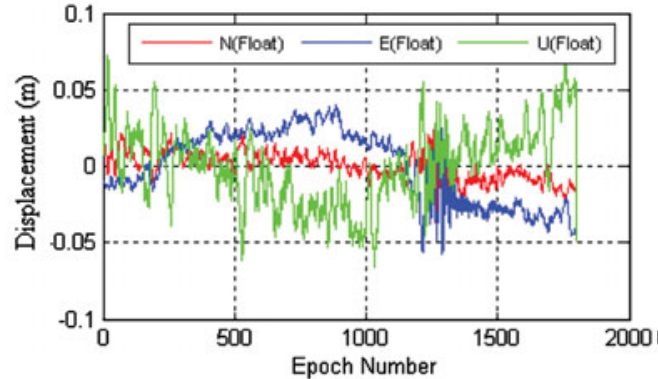

(a) PPP float solution

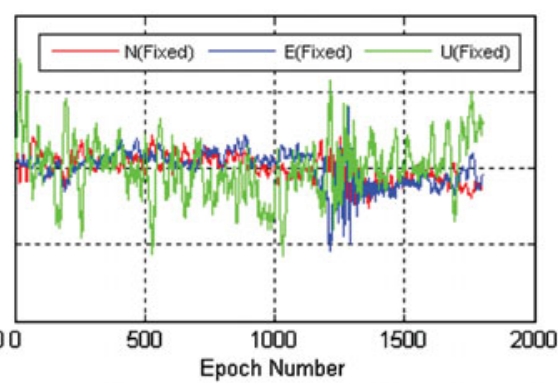

(b) PPP fixed solution

Figure 13. Displacement time series of BANA with float solution (a) and fixed solution (b).

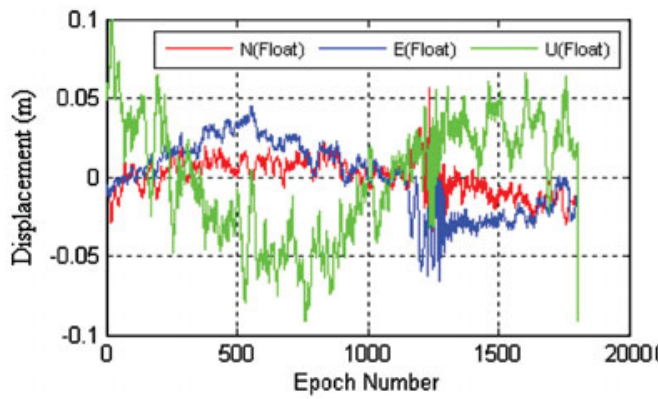

(a) PPP float solution

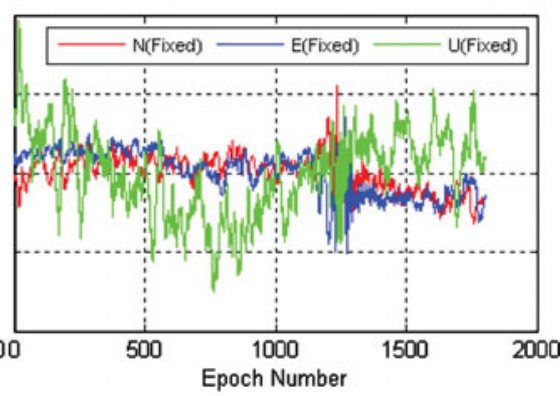

(b) PPP fixed solution

Figure 14. Displacement time series of HECU with float solution (a) and fixed solution (b).

than $2 \mathrm{~cm}$, but there are larger fluctuations in the East and vertical components with accuracies of about $5 \mathrm{~cm}$ and $10 \mathrm{~cm}$ respectively. Therefore, due to the large fluctuations of positioning results, it is not easy to extract the seismic displacement signal since it is easily hidden by noise. It can be seen from Figure 13(b) and Figure 14(b) that the displacement time series of the fixed solution is more stable in the North and East directions with an accuracy of approximately $1 \sim 2 \mathrm{~cm}$, and $5 \mathrm{~cm}$ in the vertical direction. The improvement in positioning accuracy by fixing the integer ambiguity shows that PPP can be a promising tool for co-seismic surface displacement monitoring.

6. CONCLUSIONS. We have developed a new approach for estimating Uncalibrated Fractional Offsets (UFOs) using the float estimates of Zero-Differenced (ZD) ambiguities of a reference network as observations. The ZD UFOs of receivers and satellites are estimated in an integrated adjustment which can yield the possibility of fixing integer ZD ambiguities. The estimation and fixing of ambiguities are carried out iteratively, so that the derived ZD UFOs can be of high quality. The approach can be applied to both Wide-Lane (WL) and Narrow-Lane (NL) ambiguities for the estimation of the corresponding UFOs. 
The float ZD ambiguities used in this approach can be from network or Precise Point Positioning (PPP) solutions. As the network solution is very time-consuming, the number of stations involved has to be limited, especially for real-time applications. Using the PPP solution, UFOs can be estimated flexibly from a rather dense network. A data processing procedure for providing a PPP service with Integer Ambiguity Resolution has been designed and developed for validating the new approach.

The new approach and the data processing procedure have been validated with several experimental data sets. The UFOs were estimated from reference networks of various scales and provided to users in different locations. From the results, we can conclude that UFOs estimated from the new approach are accurate and reliable enough for achieving PPP with Integer Ambiguity Resolution at a single station on the ZD level. The position and ZPD estimates are significantly improved. The accuracy of static float solutions are generally several centimetres to $10 \mathrm{~cm}$ using thirty minutes of observations, while the accuracy of fixed solutions is about $5 \mathrm{~mm}$ horizontally and about $2 \mathrm{~cm}$ in height. Improvement in the East direction is especially significant. ZPD accuracy obtained in the fixed solution is also improved by $10-50 \%$. The kinematic positioning experiment also demonstrates that a fixed solution can improve PPP accuracy significantly.

The proposed approach is also suitable for providing real-time precise positioning services.

\section{ACKNOWLEDGEMENTS}

The authors would like to thank Dr. Maorong Ge from the GeoForschungsZentrum German Research Centre for Geosciences, Potsdam. His advice and guidance were quite valuable and helpful during this research. Thanks also go to the IGS for the GPS data and products.

\section{REFERENCES}

Bisnath, S. and Gao, Y. (2007). Current State of Precise Point Positioning and Future Prospects and Limitations. Proceedings of Observing our Changing Earth, IAG Symposia 133: 615-624.

Blewitt, G. (1989). Carrier Phase Ambiguity Resolution for the Global Positioning System Applied to Geodetic Baselines up to 2,000 km. Journal of Geophysical Research, 94(B8): 10187-10203.

Collins, P., Lahaye, F., Hérous, P. and Bisnath, S. (2008). Precise Point Positioning with Ambiguity Resolution Using the Decoupled Clock Model. Proceedings of ION GNSS, GA, USA.

Dong, D., Bock, Y. (1989). Global Positioning System Network Analysis with Phase Ambiguity Resolution Applied to Crustal Deformation Studies in California. Journal of Geophysical Research, 94(B4): 3949-3966.

Gabor, M. J. and Nerem, R. S. (1999). GPS Carrier Phase Ambiguity Resolution Using Satellite-Satellite Single Difference. Proceedings of $12^{\text {th }}$ International Technical Meeting Satellite Division, Institute Navigation GPS 99, Nashville, TN, USA.

Gao, Y. and Shen, X. (2001). Improving Ambiguity Convergence in Carrier Phase-Based Precise Point Positioning. Proceedings of ION GPS-2001 pp1532-1539, Salt Lake City, USA.

Ge, M., Gendt, G., Rothacher, M., Shi, C. and Liu, J. (2008). Resolution of GPS Carrier-Phase Ambiguities in Precise Point Positioning (PPP) with Daily Observations. Journal of Geodesy 82(7): 389-399.

Geng, J., Meng, X., Dodson, A. and Teferl, F. (2010). Integer Ambiguity Resolution in Precise Point Positioning: Method Comparison. Journal of Geodesy, doi:10.1007/s00190-010-0399-x.

Hatch, R. (1996). The Promise of a Third Frequency. GPS World, 55-58.

Hofmann-Wellenhof, B., Lichtenegger, H. and Collins, J. (1992). GPS: Theory and Practice (Third edition). Wien, New York:Springer-Verlag. 
Kouba, J. and Héroux, P. (2001). Precise Point Positioning Using IGS Orbit and Clock Products. GPS Solutions, 5(2): 12-28.

Laurichesse, D., Mercier, F., Berthias, J. P. and Bijac, J. (2008). Real Time Zero-Difference Ambiguities Fixing and Absolute RTK. Proceedings of ION National Technical Meeting, San Diego, CA, US.

Li, X., Zhang, X. and Ge, M. (2010). Regional Reference Network Augmented Precise Point Positioning For Instantaneous Ambiguity Resolution. Journal of Geodesy, doi: 10.1007/s00190-010-0424-0.

Melbourne, W. G. (1985). The Case for Ranging in GPS-based Geodetic Systems. Proceedings of First International Symposium on Precise Positioning with the Global Positioning System, pp. 373-386, Rockville, MD, USA.

Teunissen, P. J. G. (1995). The Least Squares Ambiguity Decorrelation Adjustment: a Method for Fast GPS Integer Estimation. Journal of Geodesy, 70: 65-82.

Teunissen, P. J. G., Joosten, P. and Tiberius, C. C. J. M. (1999). Geometry-free Ambiguity Success Rates in Case of Partial Fixing. Proceedings of ION 55th National Technical Meeting, San Diego, CA, USA.

Teunissen, P. J. G. and Kleusberg, A. (1996). GPS for Geodesy, Volume 60 of Lecture Notes in Earth Sciences, pp. 175-217. Springer-Verlag.

Wübbena, G. (1985). Software Developments for Geodetic Positioning with GPS using TI-4100 Code and Carrier Measurements. Proceedings of First International Symposium on Precise Positioning with the Global Positioning System, pp. 403-412, Rockville, Maryland USA.

Zhang, X. and Andersen, O. B. (2006). Surface Ice Flow Velocity and Tide Retrieval of the Amery Ice Shelf Using Precise Point Positioning. Journal of Geodesy, 80(4): 171-176.

Zhang, X., Li, X. and Guo, F. (2010). Satellite Clock Estimation at $1 \mathrm{~Hz}$ for Realtime Kinematic PPP Applications. GPS Solutions. doi: 10.1007/s10291-010-0191-7.

Zumberge, J. F., Heflin, M. B., Jefferson, D. C., Watkins, M. M., Webb, F. H. (1997) Precise Point Positioning for the Efficient and Robust Analysis of GPS Data from Large Networks. Journal of Geophysical Research, 102(B3): 5005-5017. 